\title{
AVALIAÇÃO ELETROGLOTOGRÁFICA DE MULHERES DISFÔNICAS COM LESÃO DE MASSA
}

\section{Electroglottograpical evaluation of dysphonic women with mass lesions}

\author{
Aline Mansueto Mourão ${ }^{(1)}$, lara Barreto Bassi (2), Ana Cristina Côrtes Gama ${ }^{(3)}$
}

\begin{abstract}
RESUMO
Objetivo: comparar os resultados da avaliação eletroglotográfica entre mulheres adultas com laudo otorrinolaringológico de nódulos vocais e presença de fenda glótica com mulheres sem alterações laríngeas. Método: trata-se de 20 mulheres, sendo $10 \mathrm{com}$ exame otorrinolaringológico de nódulo vocal, associado à fenda glótica, e $10 \mathrm{com}$ vozes avaliadas na análise perceptivo-auditiva como neutras e avaliação otorrinolaringológica sem alteração laríngea. A faixa etária variou de 27 a 55 anos, com média de 37,60 e 36,00 para grupo estudo e controle, respectivamente. O material de voz foi a emissão habitual da vogal sustentada /a/. Foram extraídas as medidas de quociente de contato, frequência fundamental, jitter, bem como a classificação e interpretação dos tipos de ondas e presença do joelho eletroglotográfico. A análise estatística foi realizada pelo teste Exato de Fisher e T de Student. Resultados: todos os traçados foram classificados como tipo II (100\%). Não foi observada diferença na presença ou ausência de joelho entre grupos. A média do quociente de contato foi de 42,52 e 45,56 para grupo estudo e controle, respectivamente. Apesar do menor valor para o grupo estudado, não houve diferença significante entre grupos. Houve relação estatisticamente significante entre grupos no que se refere à frequência fundamental e jitter. A média da frequência fundamental foi 193,18 e 211,69 e o valor médio de jitter foi 1,21 e 2,9 para o grupo estudo e controle, respectivamente. Conclusão: mulheres com lesão de massa nas pregas vocais apresentam maiores valores de jitter e menores valores para frequência fundamental eletroglotográfica.
\end{abstract}

DESCRITORES: Voz; Disfonia; Distúrbios da voz; Fonação; Mulheres

\section{INTRODUÇÃO}

A eletroglotografia (EGG) é um método objetivo não invasivo e de simples medida, utilizado para monitorar a vibração das pregas vocais ${ }^{1-3}$. O princípio subjacente ao método foi criado por Fabre (1957) ${ }^{3}$ sendo que uma corrente elétrica pequena e de alta frequência, geralmente entre $300 \mathrm{KHz}$ e $5 \mathrm{MHz}$, é transmitida entre dois eletrodos que são colocados externamente sobre os lados da cartilagem tireóidea ao nível da laringe. Nota-se que

(1) Fonoaudióloga formada pela Universidade Federal de Minas Gerais - UFMG - Belo Horizonte (MG), Brasil.

(2) Fonoaudióloga; Mestranda em Saúde e Trabalho: Saúde Pública pela Universidade Federal de Minas Gerais UFMG - Belo Horizonte (MG), Brasil.

(3) Fonoaudióloga; Professora Associada do Departamento de Fonoaudiologia da Universidade Federal de Minas Gerais UFMG - Belo Horizonte (MG), Brasil.

Conflito de interesses: inexistente o tecido humano é um condutor elétrico razoavelmente bom quando comparado ao ar ${ }^{4} \mathrm{e}$, dessa forma, a abertura e o fechamento das pregas vocais causam variações na impedância elétrica através da laringe, tendo por resultado uma variação no fluxo entre os eletrodos ${ }^{4,5}$.

A forma da onda eletroglotográfica reflete a quantidade de impedância transversal ao nível da laringe, sendo que a impedância diminui enquanto o contato da prega vocal aumenta ${ }^{5}$. Consequentemente a onda eletroglotográfica avalia a função vocal, medindo a variação do contato da mucosa das pregas vocais no decorrer do tempo no sentido póstero-anterior e infero-superior da borda livre, durante o ciclo vibratório ${ }^{6,7}$.

A influência do grau de adução glótica sobre a onda eletroglotográfica foi analisada e interpretada por Titze (1990) ${ }^{7}$ sendo que os efeitos de (1) aumento da adução glótica; (2) convergência glótica vertical e progressiva; (3) abaulamento medial das 
superfícies das pregas vocais e (4) aumento vertical e gradual na vibração das pregas vocais têm sido discutidos. Segundo a orientação internacional, o sentido ascendente da onda eletroglotográfica indica o aumento na área de contato das pregas vocais. Um grande aumento nessa onda corresponde ao rápido fechamento das pregas vocais, seguido por um declínio gradual que está associado à separação das pregas vocais, devido à pressão abaixo da glote apresentar-se maior do que anteriormente ${ }^{5}$. Depois desse processo, as pregas vocais naturalmente retornam à sua posição de equilíbrio. A eletroglotografia tem sido amplamente utilizada para investigar funções vibratórias das pregas vocais em adultos com e sem patologias correspondentes aos distúrbios da voz ${ }^{8-10}$.

Uma medida quantitativa para obter a duração do contato das pregas vocais de um único ciclo glótico foi introduzida por Rothenberg (1992) ${ }^{8}$. Esta medida é geralmente conhecida como quociente de contato (CQEGG). Alguns autores mostraram que o quociente de contato depende da escolha do algoritmo utilizado para obter o início e o término das fases de abertura e fechamento das pregas vocais e, deve ser usado com cuidado ${ }^{2,8-10}$.

As alterações laríngeas causam desequilíbrio no funcionamento coordenado da fonação ${ }^{11}$ e quando ocorrem devido a uma lesão de massa na borda livre das pregas vocais, podem causar um fechamento glótico incompleto ou inconsistente, ocasionando escape aéreo durante a produção vocal ${ }^{4}$. 0 nódulo vocal é considerado a lesão de massa mais comum da laringe e é definido como um processo inflamatório das pregas vocais, localizado no terço médio da parte membranácea e na face subglótica, próxima à borda livre, decorrente do processo traumático pelo uso da voz ${ }^{12-14}$. A associação de nódulos e fenda triangular médio-posterior é uma constante na avaliação laríngea ${ }^{4}$. Alguns estudos mostraram diferença significante entre as medidas eletroglotográficas em mulheres com e sem nódulos vocais como, por exemplo, menor valor de quociente de contato em mulheres com tais nódulos 6,15,16. Outro estudo observou que mulheres com nódulos vocais demonstraram menor relação entre a fase de fechamento e abertura da prega vocal quando comparada ao grupo de mulheres sem alteração laríngea ${ }^{17}$. Há estudos que não observaram diferença significativa na eletroglotografia em professoras com e sem queixa vocal e/ou laríngea, porém essas não apresentavam diagnóstico laríngeo prévio ${ }^{18,19}$.

As irregularidades de vibração das pregas vocais podem ser causadas por múltiplos fatores, sendo a diferença na massa entre as pregas vocais uma delas ${ }^{20}$. A medida eletroglotográfica ajuda avaliar objetivamente a gravidade do edema ${ }^{21} \mathrm{e}$, por meio da visualização gráfica da onda eletroglotográfica pelo paciente pode, ainda, facilitar o processo terapêutico ${ }^{1}$. Para um tom de voz normal, a EGG também permite avaliar indiretamente a amplitude de contato das pregas vocais ${ }^{22}$.

Várias interpretações têm sido relatadas em relação às medidas da onda eletroglotográfica e seus parâmetros em diferentes padrões vocais com e sem alteração laríngea ${ }^{23-26}$. Dessa forma, optouse neste estudo pela utilização da avaliação eletroglotográfica em mulheres para investigar a função vocal, pois devido à configuração glótica, o sexo feminino apresenta alta prevalência de fechamento incompleto das pregas vocais quando comparado ao sexo masculino ${ }^{23-25}$ mesmo em vozes não patológicas. Além disso, percebe-se que o nódulo vocal é mais frequente em mulheres na faixa etária dos 30 aos 40 anos de idade ${ }^{27,28}$. Além disso, optouse por professoras, pois a docência requer grande demanda vocal, sendo considerada grupo de risco para os distúrbios vocais já que apresenta grandes taxas de prevalência de alteração laríngea ${ }^{18,29,30}$.

O objetivo do presente estudo foi o de comparar os resultados da avaliação eletroglotográfica de mulheres adultas com laudo otorrinolaringológico de nódulos vocais e presença de fenda glótica, com resultados da avaliação eletroglotográfica de mulheres sem alterações laríngeas.

\section{MÉTODO}

Foi realizada uma pesquisa de caráter experimental, corte transversal do tipo caso-controle no Ambulatório de Fonoaudiologia do Hospital das Clínicas (AF-HC) da Universidade Federal de Minas Gerais (UFMG). Esta casuística é composta de 20 mulheres, professoras da Rede Municipal de Ensino de Belo Horizonte. Todas foram encaminhadas pela Gerência de Saúde do Servidor e Perícia Médica da Prefeitura de Belo Horizonte para atendimento no AF-HC-UFMG. A idade variou de 27 a 55 anos, com média de 37,60 para o grupo de estudo e 36,00 para o grupo controle com desvio padrão de 8,47 e 5,25 , respectivamente.

As professoras foram convidadas a participar da pesquisa, ocasião em que foram explanados os objetivos da pesquisa e do instrumento a ser utilizado. Aquelas que concordaram assinaram um Termo de Consentimento Livre e Esclarecido. As participantes foram divididas em dois grupos: o grupo de estudo foi composto por 10 professoras, tendo como critério de inclusão o exame otorrinolaringológico de nódulo vocal e/ou espessamento da borda livre das pregas vocais e/ou lesão de massa nas pregas vocais, associado à fenda glótica. $\mathrm{O}$ 
grupo controle foi composto de 10 professoras com vozes avaliadas na análise perceptivo-auditiva como neutras sem alteração e/ou queixa vocal e avaliação otorrinolaringológica com ausência de alteração laríngea.

Foi utilizado o programa CSL da Kay Pentax ${ }^{\mathrm{TM}}$, model 6103, Lincoln Park NJ USA - módulo Electroglottography instalado no computador da marca Dell囚, modelo Optiplex GX260, com placa de som profissional marca Direct Sound $囚$, disponíveis no AF-HC-UFMG. Todos os registros de gravações eletroglotográficas das vozes foram analisados na instituição supracitada.

O material de voz coletado de cada participante foi a emissão sustentada da vogal /a/, sendo as mesmas emitidas em frequência e intensidade utilizadas habitualmente, no registro modal, de modo prolongado. Todas foram posicionadas sentadas confortavelmente de maneira a minimizar os seus movimentos durante a captação do traçado eletroglotográfico.

Verificou-se a captação da onda pelo traçado eletroglotográfico por meio da colocação simétrica de dois eletrodos, limpos e despolarizados com solução salina, de forma superficial, nas lâminas da cartilagem tireóidea ao nível das pregas vocais, conectados e diretamente digitalizados no computador. A verificação da exata localização dos eletrodos foi realizada com uma pré-testagem, na qual enquanto a participante emite a vogal /a/ sustentada, os eletrodos eram deslocados até verificar-se o melhor sinal do fluxo da corrente, ou seja, livre de interferências externas e com linha de base moderadamente estável. Com isso, utilizou-se a forma de onda eletroglotográfica mais estável e regular.

Foram extraídas as medidas eletroglotográficas de frequência fundamental (F0), índice de perturbação de frequência (jitter), e quociente de contato (CQ).

A frequência fundamental eletroglotográfica é uma medida da razão de vibração quase periódica das pregas vocais. È calculada usando a mesma fórmula descrita para medidas acústicas de F0.

O jitter eletroglotográfico representa a variabilidade ciclo a ciclo do período da frequência fundamental nos sinais de EGG. A definição de jitter eletroglotográfico é similar as equações do jitter acústico. A unidade utilizada é porcentagem.

O CQ é a medida quantitativa do grau de contato das pregas vocais durante a fonação. É representado pela razão entre o contato das pregas vocais em um determinado tempo em relação ao tempo de contato das pregas vocais em um único ciclo vibratório. É expresso em porcentagem.
Além do quociente de contato, as ondas eletroglotográficas foram analisadas qualitativamente, classificadas e interpretadas de acordo com as características da forma da onda eletroglotográfica, relacionando-as com modelos das variações geométricas da glote descritos por Titze (1990) ${ }^{7}$, a saber:

1. Pulso alargado: ocorre quando há um deslocamento uniforme de toda a borda livre em direção à linha média;

2. Pico inclinado: ocorre quando há um aumento de convergência na glote, ou seja, uma prega vocal com o ângulo mais agudo, em forma de cunha;

3. Pulso com forma abaulada: ocorre quando observamos dois joelhos no traçado, um na subida e outro na descida;

4. Pulso com forma em rampa: é produzido por uma pequena diferença no ângulo de fase entre as margens superior e inferior da borda livre das pregas vocais, modificando a forma de onda para uma imagem mais quadrangular, ou para uma imagem mais triangular, quando o ângulo de diferença entre as margens superior e inferior é grande.
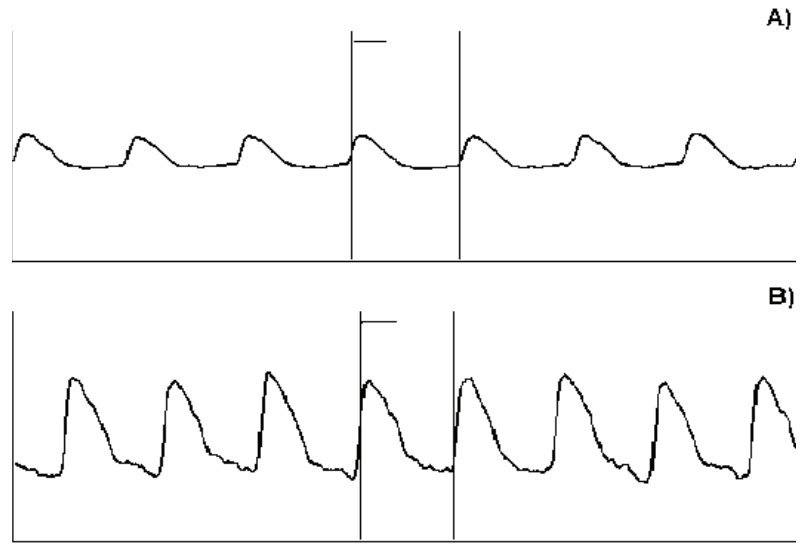

Legenda: Eixo X: amplitude; Eixo Y: tempo; Figura A) Traçado eletroglotográfico de uma mulher com diagnóstico otorrinolaringológico de nódulo nas pregas vocais; Figura B) Traçado eletroglotográfico de uma mulher com diagnóstico otorrinolariongológico de sem alteração laríngea.

Figura 1 - Forma do traçado eletroglotográfico de ambos os grupos estudados

Foi também identificada a presença ou ausência do joelho na onda eletroglotográfica que corresponde a passagem do afastamento da margem inferior da borda livre da prega vocal para a margem superior.

A pesquisa foi aprovada pelo Comitê de Ética em Pesquisa da UFMG sob o parecer ETIC 482/08. 
As respostas das professoras participantes foram organizadas, digitalizadas em programa Excel e os dados separados por parâmetros a serem analisados (quociente de contato e forma da onda eletroglotográfica). Em seguida, procedeu-se a análise descritiva das variáveis, proporções (variáveis categóricas) e medidas de tendência central e dispersão (variáveis continuas). Utilizou-se o teste Exato de Fisher para a verificação de associação entre as variáveis categóricas e o teste $T$ de Student para as variáveis contínuas. Foi considerado o nível de confiança de $95 \%$ e o programa utilizado foi o SPSS versão 17.

\section{RESULTADOS}

Foram obtidos os dados eletroglotográficos de quociente de contato, frequência fundamental, jitter e avaliação qualitativa das ondas eletroglotográficas.

Quanto à forma da onda, todos os traçados do presente estudo apresentaram, segundo os crité- rios propostos por Titze (1990) 7, "pico inclinado" (100\%), em ambos os grupos (Figura1).

A tabela 1 mostra a distribuição numérica e percentual encontrada quanto ao joelho no traçado eletroglotográfico para ambos os grupos estudados. Não observou-se diferença na presença ou ausência de joelho no traçado eletroglotográfico entre grupos.

A tabela 2 indica a média dos valores do quociente de contato encontrados para os grupos. Observa-se que, apesar do grupo de estudo apresentar menor valor para o quociente de contato, não há diferença significante quando comparado ao grupo controle.

A tabela 3 mostra a média dos valores da frequência fundamental encontrados no presente estudo. Percebe-se relação estatisticamente significante entre os grupos e menor valor da FO para o grupo de estudo.

A tabela 4 aponta a média dos valores de jitter encontrados para ambos os grupos. Observa-se diferença na medida de pertubação de frequência, sendo o maior valor para o grupo de estudo.

Tabela 1 - Distribuição numérica e percentual quanto à presença ou ausência de joelho no traçado eletroglotográfico para ambos os grupos estudados

\begin{tabular}{|c|c|c|c|c|c|}
\hline \multirow{2}{*}{ Grupo } & \multirow{2}{*}{ Amostra } & \multicolumn{3}{|c|}{ Joelho } & \multirow{2}{*}{$\mathbf{P}$} \\
\hline & & SIM & NÃO & Total & \\
\hline \multirow{2}{*}{ Caso } & $\mathrm{N}$ & 5 & 5 & 10 & \multirow{6}{*}{0,5} \\
\hline & $\%$ & $50 \%$ & $50 \%$ & $100 \%$ & \\
\hline \multirow{2}{*}{ Controle } & $\mathrm{N}$ & 7 & 3 & 10 & \\
\hline & $\%$ & $70 \%$ & $30 \%$ & $100 \%$ & \\
\hline \multirow{2}{*}{ Total } & $n$ & 12 & 8 & 20 & \\
\hline & $\%$ & $60 \%$ & $40 \%$ & $100 \%$ & \\
\hline
\end{tabular}

Legenda: N: número; \% porcentagem; P: P-valor <0,05. Teste Estatístico: Teste Exato de Fisher.

Tabela 2 - Média de valores do quociente de contato para ambos os grupos estudados

\begin{tabular}{c|c|c|c|c}
\hline \multirow{2}{*}{ Grupo } & \multirow{2}{*}{$\mathbf{N}$} & \multicolumn{2}{|c|}{ Quociente de Contato } & \multirow{2}{*}{ P } \\
\cline { 3 - 4 } & & Média & Desvio Padrão & \\
\hline Caso & 10 & 42,52 & 5,13 & \multirow{2}{*}{0,133} \\
\hline Controle & 10 & 45,56 & 3,3 & \\
\hline
\end{tabular}

Legenda: N: número de pacientes; P: P-valor <0,05. Teste Estatístico: Teste T de Student. 
Tabela 3 - Média de valores da frequência fundamental expressos em hertz para ambos os grupos estudados

\begin{tabular}{c|c|c|c|c}
\hline \multirow{2}{*}{ Grupo } & \multirow{2}{*}{$\mathbf{N}$} & \multicolumn{2}{|c|}{ Fo } & \multirow{2}{*}{ P } \\
\cline { 3 - 4 } & & Média & Desvio Padrão & \\
\hline Caso & 10 & 193,18 & 19,29 & \multirow{2}{*}{$0,028^{\star}$} \\
\hline Controle & 10 & 211,69 & 15,13 & \\
\hline
\end{tabular}

Legenda: N: número de pacientes; F0: Frequência Fundamental; P: P-valor <0,05. Teste Estatístico: Teste T de Student.

Tabela 4 - Média de valores da medida de jitter para ambos os grupos estudados

\begin{tabular}{c|c|c|c|c}
\hline \multirow{2}{*}{ Grupo } & \multirow{2}{*}{$\mathbf{N}$} & \multicolumn{2}{|c|}{ Jitter } & \multirow{2}{*}{$\mathbf{P}$} \\
\cline { 3 - 4 } & & Média & Desvio Padrão & \\
\hline Caso & 10 & 2,9 & 1,70 & \multirow{2}{*}{$\mathbf{0 , 0 0 1}^{*}$} \\
\hline Controle & 10 & 1,21 & 0,82 & \\
\hline
\end{tabular}

Legenda: N: número de pacientes; Jitter. Medida de perturbação de frequência; P: P-valor <0,05. Teste Estatístico: Teste T de Student.

\section{DISCUSSÃO}

Vários recursos de avaliação e diagnóstico dos distúrbios da voz podem ser citados como avanços tecnológicos e científicos no âmbito da Fonoaudiologia ${ }^{11}$. A eletroglotografia refere-se ao equipamento que oferece a possibilidade de estudo do que diz respeito ao contato das pregas vocais durante o ciclo glótico, sendo utilizado para avaliar a função vocal ${ }^{1-4}$. É um instrumento antigo que apresenta várias interpretações em relação às medidas da onda e seus parâmetros qualitativos ${ }^{8,16}$.

Quanto à análise dos parâmetros eletroglotográficos, no que se refere às características da forma da onda eletroglotográfica, todos os traçados do presente estudo (100\%) apresentaram , segundo Titze (1990) ${ }^{7}$, "pico inclinado".

A onda que apresenta o pico inclinado ocorre quando há um aumento de convergência glótica. Assim, o tipo de onda eletroglotográfica não diferencia os dois grupos estudados. Tal dado concorda com um estudo que também não encontrou relação estatisticamente significativa entre o tipo de onda eletroglotográfica em mulheres com e sem nódulo vocal ${ }^{6}$.

Um estudo descreve que em uma emissão vocal habitual, a eletroglotografia permite avaliar indiretamente a amplitude de contato da prega vocal durante sucessivos ciclos vibratórios, bem como as principais fases deste contato. Esta técnica exploratória permite um estudo macroscópico e microscópico do contato das pregas vocais, sendo que a análise macroscópica está preocupada com o tempo da amplitude de onda eletroglotográfica e suas variações em amplitude e frequência ${ }^{22}$.
Outro estudo teve como objetivo analisar os comportamentos vibratórios da prega vocal durante a emissão vocal em registro modal e o vocal fry em adultos, por meio do EGG. As mulheres apresentaram uma fase de abertura glótica significativamente mais longa do que os homens durante as duas fonações. Este resultado sugere que as mulheres apresentaram menor contato das pregas vocais durante as duas fonações possivelmente pela presença de fenda glótica, muito prevalente neste gênero ${ }^{26}$.

Em relação ao joelho no traçado eletroglotográfico (Tabela 1), observa-se ausência de diferença estatisticamente significante em relação à presença ou não do joelho nos grupos de mulheres com e sem alteração laríngea. Contudo, verifica-se menor ocorrência (50\%) para o grupo de estudo quando comparado ao grupo controle (70\%). O joelho eletroglotográfico indica a passagem do afastamento da margem inferior da borda livre da prega vocal para a margem superior. A ausência do joelho em mulheres com alteração laríngea pode estar associada ao espessamento na borda livre da prega vocal devido ao edema que está diretamente envolvido na configuração nodular. Tais resultados estão de acordo com estudos que encontram achados semelhantes ${ }^{6}$ e estatisticamente comprovados ${ }^{16}$.

Cabe ressaltar que nódulos e alterações na túnica mucosa localizadas no 1/3 médio das pregas vocais como cistos ou pólipos, ou até mesmo a presença de muco estão associados à alteração na fase de fechamento glótico. Dessa forma, o fechamento glótico incompleto dificulta a vibração das pregas vocais, causando interferência no sinal elétrico da onda EGG. Estudo mostra que lesões 
mais rígidas indicam uma diminuição da área de contato quando comparada às lesões maleáveis caracterizadas pela presença de edema ${ }^{16}$.

Quanto aos resultados obtidos para o quociente de contato (Tabela 2), nota-se que não houve diferença estatisticamente significante entre os grupos estudados. Apesar da média dos valores do quociente de contato no grupo de estudo e controle serem próximos (42,52 e 45,56), cabe ressaltar que mulheres com nódulo vocal apresentaram maior variação da medida quando comparadas ao grupo controle, sendo o desvio padrão de 5,13 e 3,30, respectivamente. Além disso, observa-se que o grupo controle apresenta maior valor de QC, indicando que as mulheres sem alteração laríngea apresentam maior grau de contato das pregas vocais. É esperado que o grupo de estudo tenha uma diminuição do fechamento glótico devido à alteração de massa ocasionado pelo nódulo vocal. Tais resultados confirmam os achados da literatura, aonde encontraram menor medida de QC para pacientes com lesão de massa ${ }^{6,17}$ e mulheres ${ }^{26}$.

Um estudo mostra a alta prevalência de fechamento incompleto da pregas vocais em mulheres com diferentes idades. A duração do fechamento glótico em mulheres torna-se menor com o aumento da idade. Além disso, os autores observaram aumento da duração do fechamento glótico quando há aumento na intensidade sonora e diminuição da frequência ${ }^{30}$. Tais resultados são consistentes com o estudo que avaliou o comportamento vibratório das pregas vocais correlacionando a frequência fundamental, quociente de contato com gênero e idade. No grupo das mulheres, a frequência fundamental média apresentou-se menor para o grupo de idosas quando comparada as jovens. O quociente de contato apresentou-se maior, ou seja, maior contato das pregas vocais para as idosas quando comparadas as jovens. Esse resultado pode ser devido à presença de edema nas pregas vocais após menopausa. A presença de edema nas pregas vocais poderia ter mudado a forma e viscoelasticidade dos tecidos das pregas vocais ${ }^{2}$.

Em relação à extração da frequência fundamental (Tabela 3), houve diferença estatisticamente significante entre os grupos estudados, indicando que a frequência fundamental como medida eletroglotográfica pode diferenciar os indivíduos com e sem lesão de massa. Tal resultado discorda de estudos que não encontraram diferença estatística para esse parâmetro vocal ${ }^{23}$.

É importante relatar que existe uma diferença nos dados estatísticos encontrados na frequência fundamental como medida eletroglotográfica e medida acústica da onda sonora. Estudos mostram que a frequência fundamental na EGG é de mais fácil extração do que na análise acústica da onda sonora por representar ciclos mais nítidos ${ }^{4}$. Acusticamente, quando a frequência fundamental é analisada por meio da onda sonora, esta não apresenta diferença estatisticamente significante nos grupos de mulheres com e sem nódulos vocais ${ }^{27}$.

Outro estudo teve como objetivo identificar as variações ao longo do tempo em função fonatória das mulheres com e sem nódulo vocal por meio de medidas acústicas e eletroglotográficas. As mulheres com nódulos vocais demonstraram menor relação entre a fase de fechamento e abertura da prega vocal quando comparada ao grupo controle ${ }^{17}$.

No que tange a extração da perturbação de frequência Jitter (Tabela 4), nota-se uma diferença estatisticamente significante em relação aos grupos estudados, sendo que as mulheres com alteração laríngea apresentaram uma maior pertubação do período, ciclo a ciclo, com mudanças involuntárias no período da onda eletroglotográfica.

Os valores de jitter podem ser avaliados por meio de sinais eletroglotográficos ou acústicos. Um estudo teve como objetivo determinar se estas duas técnicas de análise são medidas equivalentes para a medida de jitter. Os indivíduos foram diagnosticados como disfônicos de etiologias variadas, incluindo rouquidão persistente inespecífica, paralisia de prega vocal, nódulo vocal, úlceras de contato, pólipos, neoplasias e trauma de laringe. A análise estatística dos dados indicou que os dois instrumentos forneceram uma medida equivalente da pertubação da frequência a curto prazo ${ }^{15}$.

Outro estudo utilizou a avaliação eletroglotográfica em mulheres com diagnóstico de nódulo vocal e mulheres sem queixa e/ou alterações vocais. Foram extraídos os valores de frequência fundamental, jitter, shimmer, coeficiente de contato e índice de contato das pregas vocais. Os resultados encontraram diferenças no traçado eletroglotográfico de mulheres com nódulos vocais, sendo que este grupo apresentou maiores valores de jitter e shimmer e, menores valores de coeficiente de contato e índice de contato em relação ao grupo controle ${ }^{6}$.

Apesar do reduzido número de indivíduos avaliados, a análise do traçado eletroglotográfico nesta população poderá auxiliar na melhor compreensão do ciclo glótico de vozes com e sem alterações laríngeas.

Faz-se necessário a realização de estudos futuros em eletroglotografia a fim de se verificar a possibilidade de padronização desse instrumento e sua contribuição no processo diagnóstico das alterações laríngeas e seus conseqüentes impactos vocais. 


\section{CONCLUSÃO}

Analisando os resultados da análise eletroglotográfica em mulheres com nódulo de prega vocal e fenda glótica e em mulheres sem alteração laríngea, conclui-se que:

- Não houve diferença estatisticamente significante entre os parâmetros eletroglotográficos de tipo de onda, presença de joelho eletroglotográfico e quociente de contato.

- As mulheres com lesão de massa nas pregas vocais apresentam, na avaliação eletroglotográfica. Maiores valores de Jitter e menores valores da frequencia fundamental.

\section{ABSTRACT}

Purpose: to compare the electroglottographical evaluation results in women with otorhinolaryngological reports of vocal nodules and presence of glottal gap and in women with no larynx alteration. Method: 20 women took part in this study, 10 with otorhinolaryngological exam of vocal nodule, associated with glottal gap, and 10 with voices evaluated in the perceptive-audio analysis as neutral and electroglottographic evaluation without larynx alteration. The age group varied from 27 to 55 year old, with average of 37.60 for the study group and 36.00 for the control group. The voice material was the habitual emissions of the sustained vowel /a/. We extracted the measures of quotient of contact, fundamental frequency, jitter, as well as the sorting and interpretation of the kinds of waves and presence of electroglottographic knee. The statistical analysis has been accomplished by the Fisher Exact test and T of Student. Results: all traces were sorted as Type II (100\%). The difference in the presence or absence of knees between groups was not observed. The average of quotient of contact was 42.52 for the study group, and 45.56 for the control group. In spite of the minor value for the studied group, there has been no significant difference between groups for the fundamental frequency and jitter. The average of the fundamental frequency was 193.18 and 211.69 and, the average value of jitter was 1.21 and 2.9 for the study and control group, respectively. Conclusion: women with mass lesion in the vocal folds show higher jitter values and minor values as for the electroglottographic fundamental frequency.

KEYWORDS: Voice; Dysphonia; Voice Disorders; Phonation; Women

\section{REFERÊNCIAS}

1. Herbst CT, Howard D, Schlomicher-Their J, Salzburg. Using Electroglottographic Real-Time Feedback to Control Posterior Glottal Adduction during Phonation. J Voice. January, 2010; 24(1):72-85.

2. Ma EPM, Love AL. Electroglottographic Evaluation of Age and Gender Effects During Sustained Phonation and Connected Speech. J Voice. March, 2010; 24(2): 146-52.

3. Fabre P. Une peroce'de' e'lectrique d'inscription de l'accolement glottique au course de la phonation: Glottographie de haute frequence. Bulletin de l'Acade'mie Nationale de Me'dicine. 1957; 141:66-9 IN Rothenberg M. A multichannel electroglottograph. J Voice. 1992; 6(1): 36-43.

4. Behlau M, Madazio G, Pontes P. Disfonias Organofuncionais. In: Behlau M (Org). Voz: o livro do especialista. $1^{\circ}$ edição. Rio de Janeiro: Revinter; 2001; 296-326.
5. Baken RJ. Electroglottography. J Voice. 1992; 6:98-110.

6. Bogossian C B. Análise Eletroglotográfica em mulheres adultas disfônicas com nódulos vocais. [trabalho de conclusão de curso] São Paulo (SP): Universidade Federal de São Paulo - Escola Paulista de Medicina.1998.

7. Titze IR. Interpretation of the electroglottographic signal. J Voice. 1990; 4(1): 1-9.

8. Rothenberg M. A multichannel electroglottograph. J Voice. 1992; 6(1): 36-43.

9. Howard DM. Variation of electrolaryngographically derived closed quotient for trained and untrained adult female singers. J Voice. June, 1995; 9(2): 163-72.

10. Herbst C, Ternstrom S. A comparison of different methods to measure the EGG contact quotient. Logoped Phoniatr Vocol. 2006; 31:126-38.

11. Beber BC, Cielo CA, Siqueira MA. Lesões de Borda de pregas vocais e tempos máximos 
de Fonação. Rev. CEFAC. Jan-Mar, 2009; 11(1):134-41.

12. Braga JN, Oliveira DSF, Atherino CCT, Schot TCA, Silva JC. Nódulos vocais: análise anatomofuncional. Rev CEFAC. 2006; 8(2): 223-9.

13. Pontes $P$, Kyrillos L, Behlau M, Biase N, Pontes A. Vocal nodules and laryngeal morphology. J Voice. 2002; 16(3): 408-14.

14. Neves BMJ, Neto JG, Pontes P. Diferenciação histopatológica e imunoistoquímica das alterações epiteliais no nódulo vocal em relação aos pólipos e ao edema de laringe. Rev Bras Otorrinolaringol. Jul-Ago 2004; 70(4): 439-48.

15. Blance GR, Maves AD, Scialfa TM, Eitnier CM, Steckol KF. Comparison of electroglottographic and acoustic analysis of pitch perturbation. Laringol Head Neck Surg. 1992; 107:617-21.

16. Childers DG, Moore GP, Hicks DM, Alsaka YA. A model for vocal fold vibratory motion, contact area, and the electroglottogram. J. Acoust. Soc. Amer. 1986; 80:1309-20.

17. Hall KD. Variations across time in acoustic and eletroglottographic measures of phonatory function in women with and without vocal nodules. J. of Speech and Hear Res. 1995; 38: 783-93.

18. Lowell SY, Barkmeier-Kraemer JM, Hoit JD, Story BH. Respiratory and Laryngeal Function During Spontaneous Speaking in Teachers With Voice Disorders. J Speech Lang Hear Res. April, 2008; 51:333-49.

19. Wolfe V, Long J, Youngblood HC, Williford H, Olson MS. Vocal Parameters of Aerobic Instructors with and without Voice Problems. J. Voice. March, 2002; 16(1): 52-60.

20. Bonilha H S, Deliyski DD. Period and Glottal Width Irregularities in Vocally Normal Speakers. J Voice, 2008; 22(6) 34-40.

21. Lim J Y, Choi J N, Kim K M, Choi H S. Voice analysis of patients with diverse types of Reinke's edema and clinical use of electroglottographic measurements. Acta Oto-Laryngologica, 2006; 126: 62-9.

22. Roubeau B, Henrich N, Castellengo M. Laryngeal Vibratory Mechanisms: The Notion of Vocal Register Revisited. J. Voice, 2009; 23 (4): 425-38.

23. Guimarães I, Abberton E. Fundamental Frequency in Speakers of Portuguese for Different Voice Samples. J Voice. 2009; 19(4): 70-8.

24. Vertigan $A E$, Theodoros DG, Winkworth AL, Gibson P G. Acoustic and electroglottographic voice characteristics in chronic cough and paradoxical vocal fold movement. Folia Phoniatrica et Logopaedica. 2008; 60:210-6.

25. Silva $\vee \mathrm{O}$. Análise eletroglotográfica de diferentes tipos de vozes. [trabalho de conclusão de curso] São Paulo (SP) Centro de Estudos da Voz CECEV 1999.

26. ChenY,RobbMP, GilbertHR. Electroglottographic evaluation of gender and vowel effects during modal and vocal fry phonation. J Speech Lang. Hear Res. 2002; 45:821-9.

27. Gama ACC. Análise acústica de voz: Estudo comparativo entre mulheres com fenda triangular médio-posterior e nódulo vocal. [dissertação de mestrado] São Paulo (SP) Escola Paulista de Medicina 1997.

28. Gama ACC, Belhau MS. Estudo da constância de medidas acústicas de vogais prolongadas e consecutivas em mulheres sem queixa de voz e em mulheres com disfonia. Rev Soc Bras Fonoaudiol. 2009; 14(1):8-14.

29. Roy N, Merrill R M, Thibeault S, Gray S D, Smith E M. Voice disorders in teachers and the general population: Effects on work performance, attendance, and future career choices. J Speech Lang Hear Res. 2004; 47: 542-51.

30. Schneider B, Bigenzahn W. Influence of Glottal Closure Configuration on Vocal Efficacy in Young Normal-speaking Women. J Voice. 2002; 17 (4):468-80.
http://dx.doi.org/10.1590/S1516-18462011005000097

RECEBIDO EM: 22/12/2010

ACEITO EM: 13/04/2011

Endereço para correspondência:

Aline Mansueto Mourão

Rua Júlio Pereira da Silva 295/102, Cidade Nova

Belo Horizonte - MG

CEP: 31170-360

E-mail: alinemmourao@gmail.com 